\title{
LIE TRIPLE DERIVATIONS OF VON NEUMANN ALGEBRAS ${ }^{1}$
}

\author{
C. ROBERT MIERS
}

\begin{abstract}
A Lie triple derivation of an associative algebra $M$ is a linear map $L: M \rightarrow M$ such that

$$
L[[X, Y], Z]=[[L(X), Y], Z]+[[X, L(Y)], Z]+[[X, Y], L(Z)]
$$

for all $X, Y, Z \in M$. (Here $[X, Y]=X Y-Y X$ and $[M, M]$ is the linear subspace of $M$ generated by such terms.) We show that if $M$ is a von Neumann algebra with no central abelian summands then there exists an operator $A \in M$ such that $L(X)=[A, X]+\lambda(X)$ where $\lambda: M \rightarrow Z_{M}$ is a linear map which annihilates brackets of operators in $M$.
\end{abstract}

1. Introduction. Several authors have studied mappings between associative rings which preserve the Lie structure induced by the bracket $[X, Y]=X Y-$ $Y X$ where $X Y$ is the associative product. In particular theorems characterizing Lie isomorphisms of simple rings appear in [2] and ones characterizing Lie derivations of primitive rings appear in [5]. A parallel programme is carried out for von Neumann algebras in [6] and [7] and results entirely analogous to the ring theoretic cases are obtained even though von Neumann algebras are not, in general, simple or primitive.

A Lie triple system $M_{0}$ in an associative algebra $M$ is a subspace closed under the Lie triple product $[[X, Y], Z]$, and these structures have been studied, for example, in [3] and [4]. In [3] a theorem characterizing Lie triple system homomorphisms for certain rings is proved and in [8] the analogous theorem for von Neumann algebras appears. There does not, however, seem to be a ring theoretic analogue of the result of this paper, namely that a Lie triple derivation on a von Neumann algebra $M$ with no abelian summands, is essentially an associative derivation. The reason for this appears to be that the identities involving Lie triple derivations do not yield enough information without utilizing the analysis present in the von Neumann case.

2. Notation and preliminaries. If $M$ is an algebra over the complex field, $[M, M]$ is the linear span of all elements of the form $[X, Y]$ with $X, Y \in M$. A Lie triple derivation on a Lie triple system $M_{0}$ in $M$ is a linear map $L$ : $M_{0} \rightarrow M$ such that

Received by the editors May 11, 1977 and, in revised form, September 21, 1977.

AMS (MOS) subject classifications (1970). Primary 46L10; Secondary 16A68.

Key words and phrases. Lie triple derivation, von Neumann algebra.

'This research was partially supported by National Research Council of Canada grant A7682. The author wishes to express his gratitude to the Department of Mathematics, University of California, Los Angeles, for their hospitality during the preparation of this paper. 


$$
L[[X, Y], Z]=[[L(X), Y], Z]+[[X, L(Y)], Z]+[[X, Y], L(Z)]
$$

for all $X, Y, Z \in M_{0}$. In particular $M_{0}$ could be $M$ or $[M, M]$.

A von Neumann algebra $M$ is a weakly closed, selfadjoint algebra of operators on a complex Hilbert space $H$ containing the identity operator $I$. The set $Z_{M}=\{S \in M \mid[S, T]=0$ for all $T \in M\}$ is called the centre of $M$. If $P$ is a projection (= selfadjoint idempotent) in $M$, then $M_{P}=\{P A P \mid A \in$ $M$ \}. A projection $P$ is abelian if $M_{P}$ is an abelian algebra. If $A=A^{*} \in M$, the central core of $A$, denoted by $\underline{A}$, is defined to be $L U B\left\{S \in Z_{M} \mid S=S^{*}\right.$ $\leqslant A$ \}. The central core of a projection $P$ is the largest central projection contained in $P$. We denote by $\bar{P}$ the central carrier of a projection $P$. We use [1] as a general reference for the theory of von Neumann algebras.

3. Lie triple derivations on $M$. The main result of the paper is

THEOREM 1. Let $L: M \rightarrow M$ be a Lie triple derivation where $M$ is a von Neumann algebra with no abelian summands. There exists an operator $A \in M$ such that $L(X)=[A, X]+\lambda(X)$ where $\lambda: M \rightarrow Z_{M}$ is a linear map which annihilates brackets.

The proof of Theorem 1 proceeds essentially as in [5] except that at a few crucial points the von Neumann algebra structure of $M$ must be used. We first choose two projections in $M$ in a special way.

LemMa 1. Let $M$ be a von Neumann algebra, $P$ and $Q$ projections in $M$ with $\bar{P}=\bar{Q} \neq 0$. Let $M_{11}=\{P X P \mid X \in M\}, \quad M_{12}=\{P X Q \mid X \in M\}, \quad M_{21}=$ $\{Q X P \mid X \in M\}, M_{22}=\{Q X Q \mid X \in M\}$. If $A \in M_{i j}$ and $A X=0$ for all $X \in M_{j k}$ then $A=0$.

Proof. Case $1 . j=k$. Then either $A=A P=0$, or $A=A Q=0$.

Case 2. $j \neq k$. Suppose $A \in M_{11}$ and $A X=0$ for all $X$ in $M_{12}$. Since $\bar{P}=\bar{Q} \neq 0$ there exist projections $P_{1}, Q_{1}$ with $P_{1} \leqslant P, Q_{1} \leqslant Q$ and $P_{1} \sim Q_{1}$ via $V_{1} \in M$. That is $V_{1}^{*} V_{1}=P_{1}, V_{1} V_{1}^{*}=Q_{1}$. Then

$$
P A P_{1}=P A P P_{1}=P A P V_{1}^{*} V_{1} P_{1} P=(P A P)\left(P V_{1}^{*} Q_{1} Q\right)\left(V P_{1} P\right)=0 .
$$

If $P_{1}=P$ we are through. If not $P-P_{1} \neq 0$ so that $\left(\overline{P-P_{1}}\right) \bar{Q} \neq 0$. There exist $P_{2} \leqslant P-P_{1}$ and $Q_{2} \leqslant Q$ with $P_{2} \sim Q_{2}$. Choose a maximal collection $\left\{P_{\alpha}\right\}$ of projections in $M$ such that $P_{\alpha} \perp P_{\beta}$ if $\alpha \neq \beta, P_{\alpha} \leqslant P$, and $P A P_{\alpha}=0$. Then $P=\Sigma P_{\alpha}$ so that $P A P=A=0$.

Other cases are treated similarly.

Now let $M$ be a von Neumann algebra with no abelian summands, and let $P_{1}$ and $P_{2}$ be nonzero, orthogonal projections in $M$ with $P_{1}+P_{2}=I$, $\bar{P}_{1}=\bar{P}_{2}=I$ and $P_{1}=P_{2}=0$, (e.g. see [6, Lemma 14]). Let $M_{i j}=\left\{P_{i} A P_{j} \mid A \in\right.$ $M$ \}. Since $P_{i}=0$ we have that $M_{i i} \cap Z_{M}=\{0\}$. Let $L: M \rightarrow M$ be a Lie triple derivation.

Lemma 1. If $[X, Y] \in Z_{M}$ for $X, Y \in M$ then $[L(X), Y]+[X, L(Y)] \in$ $Z_{M}$. 
Proof. $0=L(0)=L[[X, Y], Z]=[[L(X), Y], Z]+[[X, L(Y)], Z]=$ $[[L(X), Y]+[X, L(Y)], Z]$ for all $Z \in M$.

LEMMA 2. For any idempotent $P \in M$,

$$
\begin{aligned}
P L(P) P X+X P L(P) P= & \{L(P)-L(P) P-P L(P)+2 P L(P) P\} X P \\
& +P X\{L(P)-L(P) P-P L(P)+2 P L(P) P\} .
\end{aligned}
$$

Proof. Apply $L$ to the identity $[[[[X, P], P], P], P]=[[X, P], P]$ and simplify.

Lemma 3. $L\left(P_{1}\right)=\left[P_{1}, S\right]+Z$ where $Z \in Z_{M}, S \in M$.

Proof. Similar to [5, Lemma 5].

From now on we assume $L\left(P_{1}\right) \in Z_{M}$. For, if the theorem is proved with this restriction, the general theorem can be proved by looking at $L^{\prime}=L(X)$ $-[X, S]$.

LeMma 4. If $X \in M_{i j}, i \neq j$, then $L(X) \in M_{i j}$.

Proof. If $X \in M_{12}, X=\left[\left[X, P_{1}\right], P_{1}\right]$. Let $L(X)=\Sigma_{1<i, j<2} X_{i j}$ where $X_{i j}=$ $P_{i} L(X) P_{j}$. Then $\Sigma_{1 \leqslant i, j \leqslant 2} X_{i j}=L(X)=\left[\left[L(X), P_{1}\right], P_{1}\right]=X_{12}+X_{21}$.

If $X, Y \in M_{12}$ then $[X, Y]=0$ so that $C=[L(X), Y]+[X, L(Y)] \in Z_{M}$ by Lemma 1. $X=\left[P_{1}, X\right]$ so that $[L(X), Y]=\left[L\left[P_{1}, X\right], Y\right]=C-$ $\left[\left[P_{1}, X\right], L(Y)\right]=C-L\left[\left[P_{1}, X\right], Y\right]+\left[\left[L\left(P_{1}\right), X\right], Y\right]+\left[\left[P_{1}, L(X)\right], Y\right]=$ $C+\left[\left[P_{1}, L(X)\right], Y\right]$. This implies

$$
\left[X_{12}+X_{21}, Y\right]=C+\left[\left[P_{1}, X_{12}+X_{21}\right], Y\right]=C+\left[X_{12}-X_{21}, Y\right] .
$$

Hence $\left[X_{21}, Y\right]=\frac{1}{2} C \in Z_{M}$. By [9, Theorem 1.3.1] $\left[X_{21}, Y\right]$ is a transcendental quasi-nilpotent and therefore, being central, is zero. Thus $X_{21} Y-$ $Y X_{21}=0$ for all $Y \in M_{12}$. This implies $X_{21} Y=0$ for all $Y \in M_{12}$ so $X_{21}=$ 0 .

Lemma 5. If $X \in M_{i i}$, then $L(X) \in M_{i i}+Z_{M}$.

Proof. If $X \in M_{11}, 0=\left[\left[X, P_{1}\right], P_{1}\right]$ so that $0=\left[\left[L(X), P_{1}\right], P_{1}\right]=X_{21}+$ $X_{12}$. Hence $X_{12}=X_{21}=0$. Thus $L(X)=X_{11}+X_{22} \in M_{11}+M_{22}$.

Let $X \in M_{11}, Y \in M_{22}$. Then $0=[X, Y]$ so that $[L(X), Y]+[X, L(Y)]$ $\in Z_{M}$. Let $L(X)=X_{11}+X_{22}, L(Y)=Y_{11}+Y_{22}$ with notation as above. Then $\left[X_{11}+X_{22}, Y\right]+\left[X, Y_{11}+Y_{22}\right]=\left[X_{22}, Y\right]+\left[X, Y_{11}\right]=Z \in Z_{M}$. This implies that $\left[X_{22}, Y\right]=P_{2} Z \in\left(Z_{M}\right)_{P_{2}}=Z_{M_{P_{2}}}$. Thus $\left[X_{22}, Y\right]$ is a central quasi-nilpotent in $M_{P_{2}}$ and so is zero. That is $X_{22} \in Z_{M_{P_{2}}}$. This implies $X_{22}=C P_{2}=C\left(I-P_{1}\right) \in M_{11}+Z_{M}$. Hence $L(X)=X_{11}+X_{22}=X_{11}$ $C P_{1}+C \in M_{11}+Z_{M}$.

Definition. If $X \in M_{i j}, i \neq j$, set $D(X)=L(X)$. If $X \in M_{i i}$ then $L(X)=$ $X^{\prime}+Z$ where $X^{\prime} \in M_{i i}, Z \in Z_{M}$. In this case set $D(X)=X^{\prime}$. Extend $D$ to all of $M$ by linearity and define $\lambda(X)=L(X)-D(X)$. ( $D$ is well defined since $M_{i i} \cap Z_{M}=\{0\}$.)

LeMMA 6. $\lambda: M \rightarrow Z_{M}$ is linear. 
Proof. Obvious.

LEMMA 7. If $X \in M_{i i}, Y \in M_{j k}(j \neq k)$ then $D(X Y)=D(X) Y+$ $X D(Y)$.

Proof. If $i \neq j$ then $X Y=0, D(X) Y=0$ and $X D(Y)=0$.

If $i=j$, say $X \in M_{11}, Y \in M_{12}$ then $X Y \in M_{12}$ and $D(X Y)=L(X Y)$. Now $X Y=[X, Y]=-\left[\left[P_{1}, X\right], Y\right]$. Hence $D(X Y)=-L\left[\left[P_{1}, Y\right], X\right]=$ $-\left[\left[P_{1}, L(Y)\right], X\right]-\left[\left[P_{1}, Y\right], L(X)\right]=-[L(Y), X]-[Y, L(X)]=[X, L(Y)]$ $+[L(X), Y]=[X, D(Y)]+[D(X), Y]=X D(Y)+D(X) Y$.

LEMMA 8. If $X \in M_{i i}, Y \in M_{i j}$ then $D(X Y)=X D(Y)+D(X) Y$.

Proof. Similar to [5, Lemma 11].

Lemma 9. $D(X Y X)=D(X) Y X+X D(Y) X+X Y D(X)$ for all $X \in M_{i j}$ $(i \neq j)$ and all $Y \in M$.

Proof. Exactly as in [5, Lemma 9] since the proof depends on the triple identity $2 X Y X=[[X, Y], X]$.

Lemma 10. $D$ is an associative derivation on $M$.

Proof. We need only check that $D(X Y)=D(X) Y+X D(Y)$ when $X \in$ $M_{12}, Y \in M_{21}$. Following [5, Theorem 1] we have that

$$
\begin{aligned}
\lambda[X, Y]= & D(X) Y+X D(Y)-D(X Y) \\
& +D(Y X)-D(Y) X-Y D(X) \\
= & Z \in Z_{M} .
\end{aligned}
$$

Multiplying (1) on the left by $X$ we get

$$
X D(Y X)-X D(Y) X-X Y D(X)=X Z,
$$

and on the left by $Y$ we get

$$
Y D(X) Y+Y X D(Y)-Y D(X Y)=Y Z \text {. }
$$

We have $Y X \in M_{22}, X \in M_{12}$ so $D(X Y X)=D(X) Y X+X D(Y X)$ by Lemma 7. Putting this in (2) we get $0=D(X Y X)-D(X) Y X-X D(Y) X$ $-X Y D(X)=X Z$ by Lemma 9 . Similarly (3) implies $Y Z=0$.

Now $X Z=0$ implies $|X| Z=0$ so that $|X| Z^{*}=0$. Hence $X Z^{*}=V|X| Z^{*}$ $=0$ where $X=V|X|$ is the polar decomposition. Similarly $Y Z^{*}=0$. Multiplying (1) by $Z^{*}$ we get $\{D(Y X)-D(X Y)\} Z^{*}=Z Z^{*}$. We have

$$
\begin{aligned}
D(Y X) Z^{*} & =D(Y X) P_{2} Z^{*}=D\left(Y X P_{2} Z^{*}\right)-Y X D\left(P_{2} Z^{*}\right) \\
& =-\left(Y X D\left(P_{2} Z^{*}\right)\right) .
\end{aligned}
$$

Similarly $D(X Y) Z^{*}=-X Y D\left(P_{1} Z^{*}\right)$. Hence

$$
Z Z^{*}=\{D(Y X)-D(X Y)\} Z^{*}=X Y D\left(P_{1} Z^{*}\right)-Y X D\left(P_{2} Z^{*}\right) .
$$

Thus $Z^{*} Z Z^{*}=0$. This forces $Z=0$. Now (1) implies that $D(X) Y+$ $X D(Y)-D(X Y)=-D(Y X)+D(Y) X+Y D(X)=0$ since $X \in M_{12}$, $Y \in M_{21}$. 
Corollary. $\lambda[X, Y]=0$ for all $X, Y \in M$.

Theorem 1 now follows by Theorem 1 of [10].

\section{REFERENCES}

1. J. Dixmier, Les algèbres d'operateurs dans l'espace Hilbertien, Cahiers Scientifiques, fasc. 25, Gauthier-Villars, Paris, 1969.

2. Richard A. Howland, Lie isomorphisms of derived rings of simple rings, Trans. Amer. Math. Soc. 145 (1969), 383-396.

3. N. Jacobsen and C. E. Rickart, Jordan homomorphisms of rings, Trans. Amer. Math. Soc. 69 (1950), 479-502.

4. W. G. Lister, A structure theory of Lie triple systems, Trans. Amer. Math. Soc. 72 (1952), 217-242.

5. Wallace S. Martindale III, Lie derivations of primitive rings, Michigan Math. J. 11 (1964), 183-187.

6. C. Robert Miers, Derived ring isomorphisms of von Neumann algebras, Canad. J. Math. 25 (1973), 1254-1268.

7. __ Lie derivations of von Neumann algebras, Duke Math. J. 40 (1973), 403-409.

8., Lie*-triple homomorphisms into von Neumann algebras, Proc. Amer. Math. Soc. 58 (1976), 169-172.

9. C. R. Putnam, Commutation properties of Hilbert space operators, Springer-Verlag, New York, 1967.

10. S. Sakai, Derivations of $W^{*}$-algebras, Ann. of Math. (2) 83 (1966), 273-279.

Department of Mathematics, University of California, los Angeles, California 90024

Department of Mathematics, University of Victoria, Victoria, British Columbia, Canada 